\title{
Insect Trypanosomatids: the Need to Know More
}

\author{
Sergei A Podlipaev
}

\author{
Zoological Institute, Russian Academy of Sciences, 199034 St. Petersburg, Russia
}

Of ten recognized trypanosomatid genera, only two - pathogenic Trypanosoma and Leishmania have been actively investigated for any length of time while the plant flagellates - Phytomonas - have recently begun to attract attention due to their role as agricultural parasites. The remaining genera that comprise parasites associated with insects have been largely neglected except for two or three containing popular isolates. This publication reviews current knowledge of trypanosomatids from insects.

Key words: Trypanosomatidae - insect and plant trypanosomatids - evolution

The trypanosomatid evolutionary tree (Philippe 1998, Stevens et al. 1998, 1999) has in its base the monophyletic clade of Trypanosoma, while Leishmania is situated in the crown with a range of insect flagellate congeries. Interestingly, although Trypanosoma and Leishmania are superficially close to each other from a medical point of view, they are far from each another on the evolutionary tree, and many isolates from insects and plants are spread between Trypanosoma and the crown of the tree.

\section{LEISHMANIA - SOME QUESTIONS}

From a phylogenetic point of view Leishmania is no more than one of the insect trypanosomatid lineages which have successfully colonized two classes of terrestrial vertebrates. Leishmania representatives occur in abundance in mammals and reptiles, but only two doubtful cases of Leishmania infection have been reported in birds (Molyneux \& Ashford 1983, Podlipaev 1990).

Birds serve as a host for various parasites, including many Trypanosoma species. Leishmania vectors - sand flies - can feed on birds in areas where Leishmania are abundant, for instance in Central Asia (Perfiliev 1966) and it is known that chickens are attractive for Lutzomyia sand flies in Brazil (Quinnell et al. 1992). Some data suggest that bird macrophages can kill Leishmania in vitro, but Trypanosoma parasites were also killed (Meirelles \& De Souza 1985). The type of bloodmeal also affects the proteolitic activity of sand flies and subsequent Leishmania development (Daba et al.

\footnotetext{
This work was supported by Russian Foundation of Basic Research (99-04-49-572).

Fax: +7 (812) 1140444 .

E-mail: sergei@weed.zin.ras.spb.ru

Received 13 April 2000

Accepted 15 May 2000
}

1997), and it is very likely that more factors may arrest Leishmania development and/or growth in birds or in sand flies with birds blood in their intestine. However, any explanation has to elucidate the differences between parasites - the 'elder' Trypanosoma and the 'younger' Leishmania - which allow the former to successfully colonize birds while preventing the latter from colonising these hosts. Accordingly, it is important to elucidate the basis of the differences outlined above not only for academic reasons but in order to understand the mechanisms of vertebrate resistance to parasites and, finally, to help find new therapies for leishmaniasis and trypanosomiasis.

Interestingly, except for one obscure report of Crithidia sp. in Phlebotomus and experimental infection of Phlebotomus by $C$. fasciculata (see Podlipaev 1990) Leishmania vectors are free from their own monogenetic parasites. Obviously the lack of monogenetic trypanosomatids in sand flies needs to be checked; if the absence is corroborated, it may be a confirmation of the independent origin of digeneity in Leishmania.

\section{INSECT TRYPANOSOMATIDS}

Fauna and descriptions - During the first century of trypanosomatid studies only 350 species of insects have been identified as hosts of monoxenous trypanosomatids (Wallace et al. 1983, Podlipaev 1990). It can be estimated that from more than 1,000,000 known species of insects no more than 2,000-2,500 species have been studied by parasitologists. So, only a minority of insect taxa originating from a limited number of locations have until now been examined for the presence of trypanosomatids. The vast majority of insect trypanosomatids are still to be described and relationships among them and with Trypanosoma sp. need to be established. Exploration of new regions has brought many new findings; recently flagellates were found in a range of Hemiptera in Brazil 
(Sbravate et al. 1989), in two new insect families in Russia and their known distribution was extended to Central Asia, the Far East and north to the polar circle (Podlipaev 1990, Podlipaev unpublished observation). Paradoxically, at the end of the millenium extensive areas remain as tabula rasa: nothing or very little is known about insect trypanosomatids in Southeast Asia, Australia, Japan and many other areas.

Questions appear from the beginning - from species descriptions and nomenclature. Currently, insect trypanosomatids appear to have the greatest number of formally valid descriptions that cannot be used in research. The descriptions differ greatly from each other by their quality and often it is impossible to compare them; indeed, it is sometimes impossible to establish correspondence between an old description and a newly-found parasite (Podlipaev \& Lobanov 1996). For example, from 69 described species of genus Leptomonas which could be estimated as formally valid taxa, only 15 may be viewed as reliable, with 10-15 descriptions being doubtful and the remaining 40 or so containing insufficient information to allow comparison with other findings (Podlipaev 1990).

A paradox occurs with the type species (by monotypy) of the genus Leptomonas - L. buetshlii Kent, 1880 from the nematode Tobrilus (Trilobus) gracilis. This report is the only finding of a trypanosomatid in a nematode and it has never been re-isolated. We also dissected numerous benthic nematodes, including Trilobus sp. and did not find any trypanosomatids (Podlipaev unpublished). Figures given in the published description do not allow this organism to be reliably assigned to the trypanosomatids or for it to be classified as a representative of another taxon. Latterly a flagellate parasitizing these nematodes has been shown to belong to Euglenidae (Nicoli et al. 1971), but it is impossible to be certain that it is the same organism as that originally described by Kent.

Distribution and host specificity - The important issue of host-specificity has often been treated somewhat arbitrarily. Such treatment ranges from the description of a new trypanosomatid from every insect host species to the assignment of parasites at the host family level (see Wallace 1966, Podlipaev 1990), finally resulting in the point of view that the majority of insect trypanosomatids are stenoxenous but that some of them may infect many hosts (Wallace et al. 1983).

Results obtained from DNA cross hybridization, multilocus isoenzyme electrophoresis, and random and universally primed polymerase chain reaction (PCR) indicate a very wide host specificity of insect trypanosomatids: no correspondence between natural groups of trypanosomatids (groups of distinctive genotypes) and host taxa were found. Genera, species or isolates of trypanosomatids from the insects do not coincide with species, genera and families of hosts and possibly do not even coincide with host orders. Insects from different families may have PCR-identical flagellates and parasites from hosts belonging to different orders may sometimes be genetically closer than trypanosomatids from the same host species (Podlipaev \& Bulat 1998, Bulat et al. 1999).

The distribution of trypanosomatids in insects is very irregular; in two orders, Hemiptera and Diptera, about 300 described species and undetermined trypanosomatids have been reported. A few, sporadic or unreliable findings (about 20 descriptions in total) have been described in seven other orders (see Podlipaev 1990). Such disproportion is obviously determined by the evolution history of trypanosomatids and is widely discussed in the literature (Vickerman 1994). There are not enough data to judge whether co-evolution (or co-cladogenesis) of monogenetic trypanosomatids and hosts has taken place in the order Diptera. Within the Hemiptera the low level of host specificity provides evidence of the low level of co-evolution of monogenetic trypanosomatids and their hosts, and there is no correspondence between any group of insects trypanosomatids with hemipteran taxons at any level (Podlipaev \& Bulat 1998, Bulat et al. 1999, Podlipaev \& Rokitskaya 1999).

Similar conclusions may be drawn from faunistic data. For example, in the monophyletic group of semiaquatic bugs (suborder Gerromorpha) (Andersen 1981), water-striders (family Gerridae) are infected by trypanosomatids in abundance and universally (it appears probable that they are the most commonly infected of all insect taxa); for the family Veliidae only three (unconfirmed) reports are known and Hydrometridae are free of flagellates in all regions explored (Wallace 1966, Podlipaev 1985, 1990, 1999). The mode of distribution of trypanosomatids among Hemiptera indicates that the coevolution of monoxenous parasites and their insect hosts appears unlikely. Of course, as only the minority of insect species have been investigated, this conclusion can only be regarded as preliminary.

\section{CULTURES}

In the last few decades investigation of trypanosomatids consists mostly of studies of trypanosomatid cultures. About 20 years ago 1015 cultures (isolates) were used in various laboratories (Wallace et al. 1983); at present no more than 40 isolates are commonly under study. Such a limited number of isolates is clearly insufficient to characterize the diversity of insect trypanosomatids 
and is also probably insufficient to infer the true position of species in a phylogenetic tree.

Moreover, different cultures are investigated rather unequally. The 'favourite' and most wellstudied are cultures of $C$. fasciculata and $C$. oncopelti. But, problems with these are apparent; since several Crithidia have been removed from the genus Crithidia to a monophyletic clade of symbiont bearing tryps (Du et al. 1994, Hollar et al. 1998), C. fasciculata is left as the only representative for the whole genus Crithidia on the rRNA tree. However, the figure from the original description of C. fasciculata L. Leger (1902) depicted epimastigotes among other cells - this fact casts some doubts on its identity and raises the possibility of mixed infection (Podlipaev 1990).

Similarly, there are several cultures designated as $C$. oncopelti, whose history is obscure. In one of them two independent cell populations that differ from each other by many features were discovered (Krylov et al. 1985). Latterly, it has been found that there are two isolates both named $C$. oncopelti differing from each other by the structure of rRNA genes (Du \& Chang 1994, Clark 1997, Hollar et al. 1998).

A low level of specificity and the ability of trypanosomatids to survive in an alien host (Hanson et al. 1968, Carvalho \& Deane 1974, Huppenrich et al. 1992) might enhance the probability of culturing mixed infections or a nonspecific (occasional) parasite. For example, from our data about $17 \%$ of findings in water-striders (Hemiptera: Gerridae) in Central Asia and the Caucasus are suspected to be mixed infections (Podlipaev unpublished).

Microscopic investigation of a host is far from being an ideal tool to determine the true nature of a mixed infection. For example, Blastocrithidia gerricola was identified as Blastocrithidia because of the presence of numerous (about 98\%) epimastigotes in the host bug but, simultaneously, it was supposed, from the morphology of the cells in culture and from the presence of rare promastigotes in the host, that there was a mixed infection in the insect and that an organism other than Blastocrithidia might have been isolated (Podlipaev 1985). So, in the case of B. gerricola it appears that the minor component of the mixed infection was probably isolated instead of the 'main' parasite. Molecular markers showed that the $B$. gerricola culture belonged or was very close to another genus Wallaceina (Bulat et al. 1999).

On the basis of our collection, a priori we can be more or less sure that only two isolates represent a specific infection: Leptomonas rigidus from the bug Salda littoralis, because the host inhabits a very specific biotope (it lives under material washed ashore in the upper intertidal zone of the White Sea close to the polar circle) and is ecologically isolated from all other Hemiptera in the region (Podlipaev et al. 1991); and Wallaceina brevicula, which was isolated in the spring under snow during winter adult diapause (Frolov \& Malysheva 1989).

Therefore, only the direct investigation of a culture may solve the question "what parasite was really isolated?". Of course, it must be remembered that the procedure of isolation itself and long laboratory cultivation may introduce some selective effect. If the data obtained from the morphological investigation of an original insect infection contradict those obtained from the study of a laboratory isolate it is necessary to consider the latter to determine the taxonomic position of the organism now in culture.

There is one more problem which is the opposite of that discussed above - this is the possibility of isolating representatives of different lifecycle stages in culture and then inadvertently mistaking these to be different taxa. Investigation of the Wallaceina lifecycle in the artificially infected insects demonstrated that in the host intestine the flagellates are represented by two morphologically different subpopulations that correspond to two 'strains' isolated from a laboratory culture (Malysheva \& Frolov 1995). In the absence of direct experimental investigation these strains were taken to be separate parasite species (Frolov \& Malysheva 1989); it now appears that these may in fact be different lifecycle stages of Wallaceina brevicula (Podlipaev et al. 1990).

\section{HOST-PARASITE SYSTEMS}

"Nature prefers that neither host nor parasite should be too hard on the other." M Burnet and DOWhite (1972), Natural History of Infectious Disease, p. 82.

Together, low level specificity, a high probability of nonspecific infection, and genetic and physiological variability of trypanosomatids may increase and facilitate the chances of establishing new host parasite systems. Moreover, it appears probably that not only insects but plants and other organisms may be involved in such interactions which can be more or less "occasional".

In some cases a paradox between the high level of parasite divergence in phylogenetic trees and the age of host-parasite systems which appear more recent is apparent. From published phylogenies (see Hollar \& Maslov 1997, Philippe 1998, Stevens et al. 1998) it seems that the digenetic insect/plant clade - Phytomonas - also has its roots in insect trypanosomatids. Many Hemiptera, even predatory bugs, use plants sap as a source of fluid and it 
is possible that this may be a precondition to the nonspecific transfer of flagellates from insect to plant and between plants. Fruit provides a rich medium for the multiplication and growth of insect flagellates (Conchon et al. 1989) and it is not inconceivable that such numerous opportunities for transfer may eventually result in successful adaptation to development in carbohydrate-rich plant tissues.

The monophyletic Phytomonas clade appears on the basis of its position within the rRNA phylogeny to be recent (Hollar \& Maslov 1997) and intraphloemic flagellates show considerable genetic differences from the latex parasites and from the parasites of insects (Muller at al. 1994, 1997, Bulat et al. 1999). On rRNA phylogenetic trees intraphloemic Phytomonas form the earliest branch of a monophyletic clade of plant flagellates (Marche et al. 1995, Hollar \& Maslov 1997). Phloem inhabiting Phytomonas are the causative agents of epiphytotic plant diseases of introduced plants in South America - coffee, oil palm, coconut and the ornamental plant Alpinia purpurata (Dollett 1984, Camargo 1999). The damage caused by flagellates is so high that botanists consider Phytomonas to be a natural barrier for plant naturalization (Mack 1996); interestingly, coffee disease has reached epiphytotic proportions twice, in the beginning and in the middle of the last century (Camargo et al. 1990). Intraphloemic trypanosomatids have been found in four species of South American native palms (Camargo 1999), but no trypanosomatids have been found in coconut and oil palms in their native regions or in Southeast Asia where palm agriculture is very extensive.

Thus, from these data and from the classical 'rules' of parasitology such pathogenic, non-stable host-parasite systems appear somewhat new, arising, in the case of palms, within historical times.

The unpredictable and random mode by which trypanosomatids may enter a new host are illustrated by exotic cases of trypanosomatids infecting ciliate nuclei. The most well-studied is the finding of Leptomonas ciliatorum in the macronucleus of the gastrotrich Paraholosticha sterkii (Görtz \& Dieckman 1987), where the symbiont is well-adapted to the host. Several other ciliates from various genera were experimentally infected by flagellates; in some cases attempts to infect ciliates succeded and in others it failed. Attempts to find infected ciliates in nature where they were found previously also proved negative.

The similarity in the origins of the "unexpected" host-parasite systems - intranuclear trypanosomatids/ciliates and Phytomonas/plant systems (e.g. flagellates/coffee) - is striking and the main features of host-parasite systems including the monogenetic trypanosomatids and Phytomonas are reflected in the cases mentioned above. I.e.: (1) occassionally 'choice' of the host, may be restricted to a particular genotype or clone (in a taxonomic sense - only a few representatives of any taxonomic level may serve as the hosts); (2) rapid coadaptation to a particular host resulting in creation of an obligatory host-parasite system. Trypanosomatids indicate that a high level of co-adaptation does not always mean a long time period of coevolution; (3) opportunist use of alternative hosts. Probably one such attempt resulted in the origination of Leishmania and plant trypanosomatids. And, it is possible, that recent reports about non-Leishmania and non-Trypanosoma parasites infecting humans (Dedet et al. 1995, Jimenes et al. 1997) fall into this category.

\section{SYSTEMATICS}

The taxonomic system of trypanosomatids is extremely conservative. From ten existing genera eight were described prior to 1909 , one genus in 1959 and the last one in 1990 (see Podlipaev 1990).

The heterogeneity of Crithidia, Leptomonas and Herpetomonas have been demonstrated by different methods (Kolesnikov et al. 1990, Camargo et al. 1992, Du et al. 1994, Fernandes et al. 1997, Hollar et al. 1998, Bulat et al. 1999).

The monophyly of symbiont containing trypanosomatids (Du et al. 1994; Hollar et al. 1998) is the most impressive example of the unreliability of classical morphological criteria (Hoare \& Wallace 1966). This clade includes Blastocrithidia, Herpetomonas and Crithidia species, each genus being clearly determined by cell morphology types. However, the existence of a symbiont bearing monophyletic clade clearly demonstrates that cell morphology is not appropriate as a taxonomic character for trypanosomatids and allows us to conclude that existing genera do not (and cannot) reflect the real biodiversity of trypano-somatids.

Insects trypanosomatids together with those genera possibly derived from them - Phytomonas and Leishmania - comprise a very polymorphic group. The existing ten genera clearly serve only to highlight the lack of reliable classification methods and, from another viewpoint, the lack of new isolates under study. Accordingly, current trypanosomatid systematics need to be revised. To gain a clear overview of trypanosomatid phylogeny we urgently need to increase our knowledge about the most variable group of trypanosomatids - the parasites of invertebrates and plants.

\section{ACKNOWLEDGEMENT}

To Prof. K Vickerman for valuable discussions and Dr J Stevens for help with the manuscript. 


\section{REFERENCES}

Andersen NM 1981. Adaptations, ecological diversifications, and the origin of higher taxa of semiaquatic bugs (Gerromorpha). Rostria Suppl.: 3-16.

Bulat SA, Mokrousov IV, Podlipaev SA 1999. Classification of trypanosomatids from insects and plants by the UP-PCR (universally primed OCR) technique and cross dot blot hybridization of PCR products. Europ J Protozool 35: 319-326.

Camargo EP 1999. Phytomonas and other trypanosomatid parasites of plants and fruit. Adv Parasitol 42: 29110.

Camargo EP, Kastelein P, Roitman I 1990. Trypanosomatid parasites of plants (Phytomonas). Parasitol Today 6: 22-25.

Camargo EP, Sbravate C, Teixeira MMG, Ulian SRB, Soares MBM, Affonso HT, Floeter-Winter L 1992. Ribosomal DNA restriction analysis and syntetic oligonucleotide robing in the identification of genera of lower trypanosomatids. J Parasitol 78: 40-48.

Carvalho ALM, Deane MP 1974. Trypanosomatids isolated from Zelus leucogrammus (Perty, 1834) (Hemiptera, Reduviidae), with a discussion on flagellates of insectivorous bugs. J Protozool 21: 5-8.

Clark CG 1997. Riboprinting: a tool for the study of genetic diversity in microorganisms. $J$ Eukaryotic Microbiol 44: 277-283.

Conchon I, Campaner M, Sbravate C, Camargo EP 1989. Trypanosomatids, other than Phytomonas spp. isolated and cultured from fruit. J Protozool 36: 412414.

Daba S, Mansour NS, Youssef FG, Shanbaky NM, Shehata MG, el Sawaf SM 1997. Vector-host-parasite inter-relationships in leishmaniasis. II. Influence of blood meal from natural vertebrate hosts with and without Leishmania infantum and L. major on the proteolytic activity in the gut of Phlebotomus langeroni (Diptera: Psychodidae). J Egypt Soc Parasitol 27: 639-649.

Dedet JP, Roche B, Pratlong F, Cales-Quist D, Jouannelle J, Benichou JC, Huerre M 1995. Diffuse cutaneous infection caused by a presumed monoxenous trypanosomatid in a patient infected with HIV. Trans $R$ Soc Trop Med Hyg 89: 644-646.

Dollet M 1984. Plant diseases caused by flagellate Protozoa (Phytomonas). Ann Rev Phytopathol 22: 115132.

Du Y, Chang K-P 1994. Phylogenetic geterogeneity of three Crithidia spp. Mol Biochem Parasitol 66: 171174.

Du Y, Maslov DA, Chang K-P 1994. Monophyletic origin of $\beta$-division proteobacterial endosymbionts and their coevolution with insect trypanosomatid protozoa Blastocrithidia culicis and Crithidia spp. Proc Natl Acad Sci USA 91: 8437-8441.

Fernandes O, Teixeira MMG, Sturm N, Souza MA, Camargo EP, Decrave WM, Campbell DA. 1997. Mini-exon gene sequences define six groups within the genus Crithidia. J Euk Microbiol 44: 535-539.

Frolov AO, Malysheva MN 1989. Crithidia allae sp. n. and Crithidia brevicula sp.n. (Protozoa, Trypanosomatidae) from the bug Nabis brevis.
Zoologicheskiy Zurnal 68: 5-10 (In Russian with English summary).

Görtz H-D, Dieckmann J 1987. Leptomonas ciliatorum n.sp. (Kinetoplastida, Trypanosomatidae) in the macronucleus of a Hypotrychous ciliate. J Protozool 34: 259-263.

Hanson WL, McGhee RB, DeBoe JH 1968. Experimental infection of Triatoma infestans and Rodnius prolixus with trypanosomatidae of the genera Crithidia and Blastocrithidia. J Protozool 15: 346-349.

Hollar L, Lukes J, Maslov DA 1998. Monophyly of endosymbiont containing trypanosomatids: phylogeny versus taxonomy. J Euk Microbiol 45: 293-297.

Hoare CA, Wallace FG 1966. Developmental stages of trypanosomatid flagellates: a new terminology. $\mathrm{Na}$ ture 212: 1385-1386.

Hollar L, Maslov DA 1997. A phylogenetic view on the genus Phytomonas. Mol Biochem Parasitol 89: 295299.

Hupperich K, Camargo EP, Milder R 1992. Ultrastructural study of the host-parasite relationship of trypanosomatids in the housefly. Parasitol Res 78: 48-55.

Jimenez MI, Chicharro C, Molina R, Canavate C, Alvar J 1997. Lower trypaniosomatid infecting an Hiv+ patient. Intern Workshop Molec Epidemiol Evolut Genet Pathogen Microorganisms, Montpellier, p. 60.

Kolesnikov AA, Maslov DA, Podlipaev SA 1990. Comparative restriction enzyme cleavage analysis of kinetoplast DNA from the lower trypanosomatids isolated in the North-West region of the USSR. Arch Protistenkd 138: 239-250.

Krylov MV, Podlipaev SA, Hayetsky AS, Belova LM, Frolov AO, Nijazbekova BY 1985. Is only one species present in a culture of Crithidia oncopelti (Kinetoplastmonada, Trypanosomatidae)? Zoologicheskiy Zurnal 64: 165-171 (In Russian with English summary).

Leger L 1902. Sur un flagelle parasite de l'Anopheles maculipennis. Compt Rend Soc Biol 54: 354-356.

Malysheva MN, Frolov AO 1995. The developmental cycle of Proteomonas brevicula (Trypanosomatidae) in the predatory bugs of the family Nabidae (Hemiptera). Parasitologija (St. Petersburg) 29: 289-297 (In Russian with English summary).

Marche S, Roth C, Philippe H, Dolle, M. Baltz T 1995. Characterization and detection of plant trypanosomatids by sequence analysis of the small subunit ribosomal RNA gene. Mol Biochem Parasitol 71: 15-26.

Meirelles MN, De Souza W 1985. Killing of Trypanosoma cruzi and Leishmania mexicana, and survival of Toxoplasma gondii, in chicken macrophages in vitro. J Submicrosc Cytol 17: 327-334.

Molyneux DH, Ashford RW 1983. The Biology of Trypanosoma and Leishmania, Parasites of Man and Domestic Animals, Taylor and Francis, London, xi + 294 pp.

Muller E, Gargani D, Banuls AL, Tibayrenc M, Dollet M 1997. Classification of plant trypanosomatids (Phytomonas spp.): parity between random-primer DNA typing and multilocus enzyme electrophore- 
sis. Parasitology 115: 403-409.

Muller E, Gargani D, Schaeffer V, Stevens J, FernandezBecerra C, Sanchez-Moreno M, Dollet M 1994. Variability in the phloem restricted plant trypanosomes (Phytomonas sp.) associated with wilts of cultivated crops. Isoenzyme comparison with lower trypanosomatids. European J Plant Pathology 100: 425-434.

Nicoli RM, Penaud A, Timon-David P 1971. Recherches systematiques sur les trypanosomides. 1. Le genre Nematodomonas n. gen. Bull Soc Zool France 96: 405-415.

Perfiliev P. 1966. Family Phlebotomidae. Fauna SSSR 93, Nauka publisher, Leningrad, 382 pp. (In Russian).

Philippe H 1998. Molecular phylogeny of kinetoplastids. In GH Coombs, K Vickerman, MA Sleigh, A Warren (eds), Evolutionary Relationships among Protozoa, Kluwer Academic Publishers, Dordrecht/Boston/ London, p. 195-212.

Podlipaev SA 1985. New species of lower trypanosomatids from Heteroptera families Gerridae and Nabidae: stages of their life cycles in nature and in the laboratory. Proc Zool Inst Leningrad 129: 3547 (In Russian with English summary).

Podlipaev SA 1990. Catalogue of world fauna of Trypanosomatidae (Protozoa). Proc Zool Inst Leningrad 144: 1-178.

Podlipaev SA 1999. Two new subspecies of trypanosomatids (Kinetoplastida: Trypanosomatidae), parasites of bugs (Heteroptera) from Cuba and the United States, with a discussion on trypanosomatids of water striders (Heteroptera: Gerridae). Zoosystematica Rossica 8: 1-5.

Podlipaev SA, Bulat SA 1998. Characterisation of trypanosomatids from insects and plants: UP-PCR (universally primed PCR) and cross hybridization of PCR products. Proc Zoological Inst St. Petersburg 276: $155-160$.

Podlipaev SA, Lobanov AL 1996. The employing of measure characters for discrimination of lower trypanosomatids. Parasitologija (St. Petersburg) 30 : 324-332 (In Russian with English summary).

Podlipaev SA, Rokitskaya TA 1999. Classification of isolates of insects trypanosomatids: isoenzyme analysis. Parasitologija (St. Petersburg) 33: 350-357 (In Russian with English summary).

PodlipaevSA, Frolov AO, Kolesnikov AA 1990. Proteomonas inconstans n.gen. n.sp. (Kinetoplastida: Trypanosomatidae) - a parasite of the bug Calocoris sexguttatus (Hemiptera: Miridae). Parasitologija (St. Petersburg) 24: 339-345 (In Russian with English summary).

Podlipaev SA, Malysheva MN, Kolesnikov AA 1991. Leptomonas rigidus sp. n. (Trypanosomatidae) - a parasite of Salda littoralis L. Hemiptera: Heteroptera). Acta Protozool 30: 121-127.

Quinnell RJ, Dye C, Shaw JJ 1992. Host preferences of the phlebotomine sandfly Lutzomyia longipalpis in Amazonian Brazil. Med Vet Entomol 6: 195-200.

Sbravate C, Campaner M, Camargo L, Conchon I, Teixeira MMG, Camargo EP 1989. Culture and generic identification of trypanosomatids of phytophagous Hemiptera in Brazi. J Protozool 36: 543-547.

Stevens J, Noyes H, Gibson W 1998. The evolution of trypanosomes infecting humans and primates. Mem Inst Oswaldo Cruz 93: 669-676.

Stevens JR, Noyes HA, Dover GA, Gibson WC 1999. The ancient and divergent origins of the human pathogenic trypanosomes, Trypanosoma brucei and $T$. cruzi. Parasitology 118: 107-116.

Vickerman K 1994. The evolutionary expansion of the trypanosomatid flagellates. Intern J Parasitol 24: 1317-1331.

Wallace FG 1966. The trypanosomatid parasites of insects and arachnids. Exp Parasitol 18: 124-193.

Wallace FG, Camargo EP, McGhee RB, Roitman I 1983. Guidelines for the description of new species of lower trypanosomatids. J Protozool 30: 308-313. 